\title{
EL CLIMA: FACTOR DE DIFERENCIACIÓN ESPACIAL. DIVISIONES REGIONALES DEL MUNDO DESDE LA ANTIGÜEDAD AL S. XVIII
}

\author{
Jorge Olcina Cantos
}

\section{RESUMEN}

El clima ha sido empleado, desde la antiguedad, por diversos autores, como elemento de diferenciación espacial. Climatólogos, meteorólogos, botánicos, agrónomos, entre otros, han elaborado, variadas divisiones regionales del clima que forman lo esencial de la climatología regional. Pero además, numerosos geógrafos han destacado el papel fundamental que cumple el clima para explicar repartos espaciales de sociedades y modos de vida e incluso, algún autor, se ha aventurado a esbozar divisiones regionales del mundo en función del factor climático. Unas y otras ofrecen unidades diversas que se dan en el espacio atendiendo a un criterio físico; son, por tanto, regiones de cariz natural existentes en la superficie terrestre.

Palabras clave: Clima, región natural, región climáticas, divisiones regionales del mundo.

\begin{abstract}
Climate has been employed, from the antiquity, by diverse authors, like spacial differentiation element. Climatologist, meteorologist, botanists, agromists, among other, have worked on, varied regional divisions of climate that form the essential thing of regional climatology. But besides, numerous geographers have emphasized the fundamental role climate carry out to explain spacial distributions of societies and ways of life and even, some author, a bit too much to sketch world regional divisions, depending on climatic factor. Both lots offer diverse unities to physical criterious; so they are regions with a natural aspect existing in the terrestrial surface.
\end{abstract}

Key words: climate, natural region, climatic region, world regional divisions. 


\section{Introducción. Región natural, región climática. Escalas de análisis}

No existe en Geografía problema tan importante como el de las diferenciaciones espaciales. En efecto, desde antiguo ha preocupado la explicación de las diferencias existentes entre unas y otras partes de la superficie terrestre. La descripción de estas particularidades y la elaboración de criterios necesarios para entender su distribución espacial ha sido tarea común de la geografía desde época griega. Con el avance del s. XIX, que inaugura la llamada etapa científica del pensamiento geográfico, la aparición de sucesivos paradigmas y los enfrentamientos acalorados que ha supuesto su desarrollo entre los seguidores de unas y otras corrientes no han hecho sino enmascarar, en no pocas ocasiones, el objetivo básico de la ciencia geográfica: la explicación de las relaciones entre el medio y el hombre.

En la interpretación de estas relaciones y sus diferencias en el espacio terrestre, el factor climático ha sido utilizado, por diversos autores — geógrafos y no geógrafos-, como elemento determinante para la elaboración de divisiones regionales del mundo. En la descripción de las diversas unidades espaciales (continentes, regiones, provincias, ...) el clima ha gozado de tratamiento preferente al considerar que sus rasgos básicos resultan esenciales para comprender el reparto de masas forestales, especies animales, cultivos agrícolas e incluso poblaciones humanas. Así pues, a la hora de explicar diferencias espaciales, el clima de un territorio ha preocupado:

a) Como factor intrínseco, es decir, descripción de las diversas características básicas que presentan sus elementos - temperaturas, precipitación, humedad, presión, vientosen unos y otros espacios (clasificaciones climáticas) y,

b) Como factor condicionante de la aparición de diferencias en el medio geográfico (divisiones regionales del mundo basadas en el clima).

Interesa destacar, pues, que la preocupación por el estudio del clima no es sólo labor de climatólogos o meteorólogos plasmada en la elaboración de variadas divisiones regionales del clima desde el s. XIX, sino que han sido numerosos los geógrafos que, desde la antigüedad clásica, han destacado el papel fundamental que cumple el clima para explicar repartos espaciales de sociedades y modos de vida e incluso, algún autor, se ha aventurado a esbozar divisiones regionales del mundo.

Así pues, en el amplio catálogo de obras destinadas a presentar divisiones del planeta en función del factor climático encontraremos:

- por un lado, clasificaciones realizadas desde la disciplina climática, sensu stricto, que integran lo esencial de la rama regional de la climatología (climatología regional). Son, pues, sistemas regionales establecidos en función de las propiedades que presentan los elementos (en este caso, climáticos), objeto de clasificación.

- por otro, divisiones regionales del mundo realizadas por geógrafos (desde la perspectiva regional o de la geografía humana) que han visto en el elemento climático el criterio esencial para establecer peculiaridades regionales (geografía regional). Se trata de divisiones realizadas teniendo en cuenta no tanto las propiedades de los elementos sino las relaciones entre ellos.

Unas y otras ofrecen, en suma, unidades diversas que se dan en el espacio atendiendo a un criterio físico; son por tanto regiones de cariz natural existentes en la superficie terrestre. 
El interés por el establecimiento de divisiones planetarias en función de elementos del medio físico (regiones naturales), ha preocupado a la ciencia geográfica desde el s. XVIII. Se suele atribuir a Ph. Buache la paternidad de las «regiones naturales» quien, en su «Ensai de Géographie Phisique», utiliza un elemento natural como unidad de diferenciación del espacio: las cuencas hidrográficas. Al margen de las críticas que recibió su método de estudio y de la existencia de antecedentes del mismo ${ }^{1}$, por vez primera, se explica la diversidad entre regiones planetarias sin recurrir a criterios políticos-administrativos, comunes desde época medieval. No obstante, si hubiera que legitimar la paternidad de las descripciones del mundo en virtud de criterios naturales habría que mencionar a los geógrafos del mundo heleno ${ }^{2}$. Ellos son los primeros que nos presentan divisiones del mundo habitable y del mundo conocido - oikumene - basadas en criterio climático: diferencias térmicas y de insolación (vid. infra).

La región natural se presenta como una entidad de análisis de lógica natural perceptible en el mundo real. Este concepto y la categorización del espacio terrestre a que ha dado lugar ha experimentado profunda evolución ${ }^{3}$ desde sus primeras manifestaciones a finales del s. XVIII. Así, las obras descriptivas de Bauche, Bausching y Catterer son intentos tempranos de conferir a la descripción del mundo un nuevo rumbo, mediante la inclusión de divisiones naturales. La obra de Humbolt constituirá un nuevo revulsivo para el estudio del mundo con perspectiva natural al considerar la descripción de los fenómenos naturales el motivo esencial de los estudios geográficos. El evolucionismo de la segunda mitad del s. XIX otorgará renovado impulso a las descripciones espaciales de base naturalista; por un lado, surge el concepto de paisaje cuyo análisis y descripción abrirá una próspera tendencia dentro de la geografía abierta a las tendencias de las ciencias naturales y donde se valorará la acción del hombre como condicionantes de la ocupación y explotación del espacio natural. En nuestro país, este impulso naturalista encuentra en los trabajos de Hernández Pacheco y Dantín Cereceda, a comienzos de siglo, las aportaciones más relevantes ${ }^{4}$. Por otra parte, a finales del s. XIX se desarrollan las concepciones deterministas del medio que defienden la primacía del medio sobre el hombre relegando su acción a una. Este sesgo determinista se mantendrá, sin embargo, en obras publicadas a mediados de nuestro siglo.

1 En el s. XVI, el español Martín Fernández de Enciso en su Summa de Geographia que trata de todas las partidas et provincias del Mundo, en especial de las Indias (1519), realiza el primer esbozo de división de regiones naturales según las cuencas hidrográficas.

2 En mi opinión, resulta poco apropiada la tradicional división del pensamiento geográfico en dos grandes etapas: precientífica y científica; esta última inaugurada por Humbolt y Ritter con el avance del s. XIX. Es cierto que en la larga etapa anterior — ¿precientífica?-, no hay una sistematización precisa, no hay paradigmas, hay carencia de aparataje y de mediciones exactas, pero hay preocupación por entender el mundo conocido y desconocido, hay inquietud por calibrar sus dimensiones, por medir distancias, en definitiva, hay explicación de hechos geográficos. La evolución del pensamiento científico (geográfico, en nuestro caso) es una lucha por mejorar los conocimientos de épocas precedentes, es, en suma, un avance constante de las inquietudes del hombre. En las distintas época históricas, el objetivo final ha sido idéntico: ensanchar el campo del conocimiento humano; lo que ha variado ha sido la posibilidad de disponer de medios técnicos cada vez más precisos lo que ha posibilitado, a su vez, la aparición de nuevos y paradigmas. El grado de cientifismo en geografía no debe establecerse en función de mejoras técnicas o de paradigmas, sino de la valoración de la calidad de los trabajos dedicados a explicar lo esencial: las relaciones entre el medio y el hombre.

3 Vid. Vilá Valentí, J. (1980) «El concepto de región» en La Región y la Geografía Española, Asociación de Geógrafos Españoles, Valladolid, pp. 13-34; Sanz Herráiz, C. (1980) «Significado de la región natural en la actualidad» en La Región y la Geografía Española, Asociación de Geógrafos Españoles, Valladolid, pp. 35-51; Castillo Requena, J. M. (1991/92) «La Región natural, un concepto en evolución» en Paralelo 37º n $^{\circ}$ 14-15, pp. 21-32.

4 Vid. Hernández Pacheco, E. (1955) Fisiografía del Solar Hispano. Real Academia de Ciencias Exactas, Físicas y Naturales, Madrid, 2 tomos (655+793 p.) y Gómez Mendoza, J. y Ortega Cantero, N. (1992) «Coordenadas del conocimiento naturalista y geográfico en España» en Naturalismo y Geografía en España. (Gómez Mendoza, J. y Ortega Cantero, N. dirs.), Fundación Banco Exterior. Colección Investigaciones, Madrid, pp. 9-77. 
A finales de los años sesenta y comienzos de los setenta del s. XX, la ciencia (o mejor, la didáctica) geográfica verá surgir un interesante intento de análisis de la superficie terrrestre con base natural: la geografía zonal. Basada ésta en la delimitación de grandes bandas o franjas del globo en virtud de la diferente recepción energética y por tanto con rasgos térmicos contrastados. Se rescata así la vieja tradición griega de división del mundo habitable en zonas en un intento didáctico de servir de eslabón entre los estudios generales y las descripciones regionales. Hay que señalar, por último, la renovación sufrida en los últimos años por el concepto de «medio natural» ${ }^{5}$ que ha servido a Demangeon para presentar una división de la superficie del planeta basada en el grado de antropización de los medios naturales.

Los enfoques y renovaciones que ha experimentado la región natural con la evolución del pensamiento geográfico no son sino maneras de entender la distribución de elementos de la superficie terrestre mediante un criterio natural donde el hombre juega un papel más o menos destacado, según unas u otras concepciones.

Se presenta a continuación un estudio de las divisiones del mundo realizadas en función de la influencia del factor climático en la vida de las sociedades, valorando la importancia que éste ha tenido para los geógrafos en sus intentos de interpretar la distribución de los hechos en la superficie terrestre.

\subsection{Escalas de análisis en las clasificaciones de base climática}

Previo al análisis de la evolución del pensamiento geográfico en la interpretación de la influencia del clima como elemento de división de unidades espaciales, es necesario detenerse en la cuestión de las escalas de trabajo manejadas tradicionalmente en las clasificaciones de base climática. El estudio de los climas del mundo, a distintas escalas, se ha servido de una serie de términos y expresiones de criterio espacial diverso que en ocasiones provocan confusión terminológica y problemas de comparación entre diferentes clasificaciones. Así, se habla de ámbitos, zonas, franjas, dominios, husos, pisos, tipos, regiones, provincias, topoclimas, microclimas, entre otras.

Según la extensión territorial a la que aluden y los mecanismos atmosféricos que entran en juego, puede hablarse de:

1. ÁMBITO: Sector planetario partícipe de un mismo balance energético. Así tendremos:

a) Ámbito intertropical con excedente calorífico entre el Ecuador y los $30^{\circ}$ de latitud norte y sur.

b) Latitudes medias y altas con déficit energético desde los $40^{\circ}$ hasta el Polo.

Entre ambas se situaría (entre los $30-40^{\circ}$ de latitud) una zona de «teórico» equilibrio calorífico, partícipe de los mecanismos de reajuste de energía que tienen lugar en el sistema atmosférico entre los dos ámbitos señalados.

La división entre los distintos ámbitos responde a diferencias de energía calorífica neta (entradas y salidas radiativas). Los límites entre unos y otros se plasman cartográficamente mediante líneas de paralelo (latitud). Se suele equiparar esta división en ámbitos basada en el balance radiativo, con las 3 «zonas térmicas» del planeta señaladas ya por los griegos en la antigüedad (vid. infra), sin embargo en esta última, la zona templada ocupa un espacio

5 Vid. Demangeon, J. (1989) Los medios «naturales» del globo. Ed. Masson, Barcelona, pp. 9-14. 
mayor que el atribuido aquí a la franja de equilibrio calorífico, en virtud de la diversa plasmación territorial de que disfrutan, en una y otra, las diferencias de radiación y temperatura, respectivamente. Las zonas térmicas establecidas por los griegos estaban fundadas en la diferente recepción calorífica solar y en criterios de habitabilidad de las diferentes zonas; de ahí que las «zonas térmicas» del planeta utilizasen, ya desde entonces, las llamadas «líneas cartográficas mayores», es decir, línea ecuatorial, trópicos y círculos polares.

2. ZONA, FRANJA o BANDA: Sector planetario afectado por idéntico movimiento de vientos, sistema de presión y ubicación de hogares de masas de aire de similares características. Desde el Ecuador al Polo se distinguen:

- zona de convergencia intertropical

- banda de los alisios

- franja de altas presiones subtropicales

- zona de circulación general del oeste

- zona polar o fría

Se trata, pues, de divisiones establecidas en virtud de las diferencias que presenta, a gran escala, la circulación atmosférica planetaria y cuyo límite viene asimismo establecido por líneas de paralelo (latitud) ${ }^{6}$. Si bien, el carácter fluctuante, a lo largo del año, de las masas de aire y de los sistemas de presión y vientos resta rigidez a la ubicación geográfica de estos límites.

3. DOMINIO: Sector planetario inserto en una franja afectado por similar régimen térmico y/o de lluvias. Desde el Ecuador al Polo se pueden distinguir:

- dominio ecuatorial

- dominio tropical (y dentro de él la variedad de dominio monzónico)

- dominio subtropical

- dominio desértico

- dominio templado,

- dominio templado-frío

- dominio frío

- dominio glaciar

Los límites de los dominios climáticos vienen marcados por la latitud y la longitud geográfica.

4. REGIÓN CLIMÁTICA: Sector planetario, inserto en un dominio climático, donde la circulación atmosférica y los elementos climáticos adquieren rasgos peculiares en función de la influencia marítima, ubicación de relieves, disposición de líneas de costa. Así, se distinguen, entre otras, de:

6 De esta misma opinión participa el prof. Orlando Ribeiro, quien afirma que las grandes ZONAS terrestres «marcan la circulación de masas de aire, el régimen de las aguas, la fisonomía de la vegetación y las condiciones generales de la vida humana en los niveles inferiores de civilización. Las ZONAS o franjas están dispuestas en el sentido de los paralelos por causas planetarias». (Vid. Ribeiro, O. (1987), Introduçao ao estudo da geografia regional, Ediçoes Joao Sá da Costa, Lisboa, pp. 40-42). 
- región de clima mediterráneo

- región de clima oceánico

- región de clima continental frío

- región de clima tropical seco...

Las regiones climáticas vienen delimitadas por criterios de latitud, longitud y altitud. En opinión de Pedelaborde (1982), los climas regionales se pueden considerar «facies geográficas» de las zonas climáticas. Manejando un criterio dimensional, siempre discutible, suele hablarse de «región climática» para un ámbito comprendido entre 1.000 y 2.000 $\mathrm{Km}^{2}$. En 1937, W.G. Kendrew en su obra «Climates of the Continents» indicó la estrecha relación existente entre las regiones climáticas y las diferencias de vegetación en el paisaje; de ahí la existencia, desde los trabajos de W. Köppen y Suppan, de numerosas clasificaciones climáticas (biogeográficas) en las que las diferentes regiones vienen denominadas en atención al tipo vegetal dominante (región tipo tundra, región tipo estepario, región tipo taiga, etc.). No obstante, estas divisiones en grandes unidades de paisaje vegetal vendrían a corresponder en mayor medida a los dominios que a las regiones climáticas. Clasificaciones de regiones (y provincias) climáticas son, en su mayor parte, las elaboradas por la escuela francesa de climatología desde Martonne y que pueden ser denominadas, con propiedad, «geográficas», puesto que asignan un nombre «regional» característico a los tipos climáticos insertos en las distintas zonas y dominios planetarios.

5. PROVINCIA CLIMÁTICA: Sector planetario, inserto en una región climática donde los rasgos del medio físico imponen particulares condiciones en los elementos del clima, permitiendo individualizar en dicho territorio unidades de extensión menor pero partícipes de los rasgos generales de la región en que se inserta. Un ejemplo vendría dado por la matización en la región mediterránea entre las provincias climáticas de Cataluña o de la Provenza y los sectores jordanos y del sureste de la Península Ibérica, asimismo mediterráneos. En ambos la «sequía» estival, aunque de intensidad diversa, es marcada pero se dan significativas diferencias estacionales de precipitación y de temperaturas. Viers (1981) dentro de lo que denomina «dominio» mediterráneo (región mediterránea) distingue los climas heleno, portugués y californiano que serían, en efecto, provincias climáticas dentro de aquella región.

6. CLIMA LOCAL: Sector planetario, inserto en una provincia climática, partícipe de condiciones climáticas particulares en función de la altitud, la exposición o la presencia de masas vegetales espesas (p.e. hayedos). En ocasiones las modificaciones introducidas por el hombre (p.e. construcción de grandes embalses) pueden favorecer la aparición de un clima local al modificar los registros elementos climáticos preexistentes (sobre todo, temperaturas y humedad). Siguiendo criterio dimensional hablaríamos de clima local para un ámbito comprendido en un radio de $10 \mathrm{Km}$. Una categoría propia dentro de éstos constituyen los llamados «climas urbanos».

7. MICROCLIMA: Sector terrestre de reducidas dimensiones partícipe de unos rasgos climáticos particulares en relación al entorno en que se inserta debido a condiciones naturales particulares (cuevas o abrigos) a modificaciones artificiales introducidas por el hombre (invernaderos). Hablaríamos de microclima para un sector comprendido en un radio de $100 \mathrm{~m}$. a $1 \mathrm{~km}$.

Estas dos últimas categorías (clima local y microclima) suelen manejarse como sinónimas, de hecho, el propio origen del término «clima local» deriva del término alemán 
Kleinklima (clima de pequeña escala), muy ambiguo en la precisión de límites espaciales. A finales de los años veinte del presente siglo, R. Geiger, fundador de los estudios microclimáticos, distingue entre Mikroklima o microclima orográfico y Kleinklima, manejados en la actualidad como equivalentes a topoclima y mesoclima. Desde entonces han sido variadas las definiciones de clima local y microclima así como los criterios dimensionales utilizados; Landsberg define el clima local como el clima cuya escala está comprendida entre el clima «puntual» (microclima) y el clima regional; para Mörikofer la extensión horizontal de un clima local esta comprendida entre 100 y 1.000 m.; Flohn y Barry aumentan este límite y definen el clima local como el comprendido entre $100 \mathrm{~m}$. y $10 \mathrm{Km}$. Yoshino, en su trabajo sobre «Climas de áreas pequeñas» (1975) completa esta dimensión horizontal con una escala vertical (entre $10 \mathrm{~cm}$. y $1 \mathrm{~km}$.).

A estas categorías hay que añadir el PISO CLIMÁTICO expresión manejada fundamentalmente en los países iberoamericanos para matizar la influencia de la altitud en una región climática; expresión equivalente a lo que sería una provincia climática definida únicamente por criterios de altitud. Recordemos que esta terminología encuentra su origen en las expresiones «tierra cálida» (1.000-2.000 m.), «tierra templada» (2.000-3.000 m.) y «tierra fría» (por encima de los $3.000 \mathrm{~m}$.) utilizada ya por diferentes cronistas de Indias (J. de Acosta), que recogieron la tradición indígena, y ha sido mantenida por diversos tratadistas hasta la actualidad (A. Miller). A gran escala, las modificaciones que introducen los grandes relieves planetarios ${ }^{7}$ en los distintos dominios y regiones climáticas otorgan, sin duda, categoría climática específica a los climas de montaña dentro de las clasificaciones climáticas y así se refleja en muchas de ellas (p.e. Koppen, Strahler). En montaña media o pequeña, los relieves imponen la aparición de provincias climáticas y de climas locales dentro de un marco climático regional ${ }^{8}$.

La sistematización aquí presentada pretende ser una propuesta de clasificación de los distintos niveles de estudio que suelen manejar los trabajos climáticos; propuesta carente de pretensiones universalistas pues, como se ha señalado, la libertad de catalogación y la carencia de un criterio general válido para cualquier división del planeta en unidades naturales, es la norma común en la bibliografía consultada. Así por ejemplo Peguy (1970) y Hufty (1984), siguiendo un criterio dimensional hablan respectivamente de Áreas que se componen de Zonas y Husos (vastas unidades continentales de orientación norte-sur, por ejemplo las fachadas occidentales de un continente), Provincias, Regiones, Estaciones (clima local) y Microclimas. A la Región climática se le otorga un sentido de región geográfica a la manera francesa vidaliana, disfrutando, por tanto de una dimensión espacial menor que la considerada en nuestra propuesta, siendo la Provincia climática la unidad espacial que correspondería con la región climática tal cual se considera en la presente propuesta, es decir, con un sentido de región natural. La misma consideración merecen estas dos categorías (provincia y región) en la obra de Estienne y Godard (1970), quienes a su vez matizan las variedades climáticas de gran escala distinguiendo entre clima local, topoclima (hayedo, clima urbano) y microclima. Pagney (1982) distingue entre zonas, regiones climáticas, climas locales y microclimas e indica que la distribución de climas

7 Estos relieves suelen recibir la denominación de «relieves de escala sinóptica» por que, al actuar sobre los mecanismos meteorológicos en la mesoscala, tienen capacidad de transformar situaciones sinópticas (p.e. procesos de ciclogénesis, ondas de montaña), además de modificar los valores de los elementos del tiempo asociados a una situación atmosférica.

8 El estudio de los climas de montaña media y pequeña es una de las lagunas con que aún hoy cuentan los trabajos climáticos. La ausencia de observatorios de montaña y, la dificultad de realizar mediciones diarias mediante trabajos de campo a lo largo de una serie suficiente de años, condicionan la elaboración de síntesis precisas sobre climas de montaña tan necesarias para completar el mosaico climático a escala regional. 


\section{Tabla I}

ESCALAS DEL CLIMA Y SU CORRESPONDENCIA CON LOS FENÓMENOS METEOROLÓGICOS SEGÚN YOSHINO (1975)

\begin{tabular}{|c|c|c|c|c|}
\hline CLIMA & $\begin{array}{c}\text { EXTENSIÓN } \\
\text { HORIZONTAL }\end{array}$ & $\begin{array}{c}\text { EXTENSIÓN } \\
\text { VERTICAL }\end{array}$ & $\begin{array}{c}\text { CLIMA } \\
\text { REPRESENTATIVO }\end{array}$ & $\begin{array}{l}\text { DURACIÓN DE LOS } \\
\text { FENÓMENOS } \\
\text { METEOROLÓGICOS }\end{array}$ \\
\hline Microclima & $10^{-2}-10^{2} \mathrm{~m}$ & $10^{-2}-10 \mathrm{~m}$ & $\begin{array}{l}\text { Clima de un } \\
\text { invernadero }\end{array}$ & hasta 10 segundos \\
\hline Clima Local & $10^{2}-10^{4} \mathrm{~m}$ & $10-10^{3} \mathrm{~m}$ & $\begin{array}{l}\text { Clima de una } \\
\text { ladera }\end{array}$ & 10 seg-2,5 horas \\
\hline Mesoclima & $10^{3}-\left(2 \cdot 10^{5}\right) \mathrm{m}$ & $10-\left(6 \cdot 10^{3}\right) \mathrm{m}$ & $\begin{array}{l}\text { Clima de una } \\
\text { cuenca }\end{array}$ & 2,5 horas-1 día \\
\hline Macroclima & $\left(2 \cdot 10^{5}\right)-\left(5 \cdot 10^{7}\right) \mathrm{m}$ & $10-10^{5} \mathrm{~m}$ & $\begin{array}{l}\text { Zona o dominio } \\
\text { climática }\end{array}$ & 1 día-11 días \\
\hline
\end{tabular}

Fuente: Yoshino, M (1975) Climate in a Small Area. Tokyo Universituy Press. Tokyo.

regionales depende de factores zonales, factores geográficos y factores azonales. Los primeros comprenden los aspectos cósmicos y planetarios que imponen el boceto zonal de los climas; los rasgos geográficos de una territorio matizan, por su parte, la existencia de climas regionales, locales y microclimas; por último, los factores azonales que condicionan la aparición de variedades continentalizadas en los climas regionales.

Otros autores (p.e. Miler, 1975), adoptando una postura más ecléctica, no matizan categorías dimensionales y hablan directamente de «climas» o «tipos climáticos», que por lo demás suelen corresponder a los dominios y regiones de nuestra propuesta. Por su parte, Yoshino (1975) ofrece una catalogación de escalas climáticas en relación con los fenómenos meteorológicos que en ellas tienen lugar (vid. tabla I). Excesivamente rígidos resultan los límites espacio-temporales que presenta el autor y, en particular, es muy discutible la gradación de la duración cronológica de los fenómenos atmosféricos en las distintas escalas de trabajo.

La determinación de dimensiones superficiales y la ubicación de límites precisos entre unidades de análisis resulta complejo y depende siempre del criterio elegido. La subjetividad es, pues, una característica común en las cuestiones de escala dentro de la geografía regional. O. Ribeiro (1987) indica, por ejemplo, que, en catalogaciones de base natural, sólo se puede hablar de límites lineales cuando el criterio de diferenciación espacial son relieves (facies) o suelos mientras que es más ajustado hablar de fajas de transición cuando el factor de diferenciación es el clima y la vegetación. Así, Daniel Faucher, en 1941, afirmaba que «la división climática de una territorio permanece casi siempre cargada de indecisiones: los márgenes cabalgan unos sobre otros»?.

Por otra parte, los intentos de comparación entre clasificaciones de unidades llevadas a cabo desde distintas disciplinas de estudio del medio natural (clima, relieve, vegetación, cursos fluviales, paisajes), sin negar su utilidad, están llamados al fracaso puesto que la libertad de elección de criterio y su correspondencia escalar es norma común entre los

9 Vid. Faucher, D. (1941) «De los países a las regiones» en Bulletin de l'Universite et de l'Academie de Toulouse, 8, pp. 285-301 (en Gómez Mendoza, J. et alt. (1988) El pensamiento geográfico, Alianza Editorial, Madrid, pp. 280-288). 
diversos autores. Orlando Ribeiro (1987) señala que las áreas (ámbitos), zonas y dominios no pueden servir de marco para la geografía regional. En este sentido, sería necesario distinguir entre unidades básicas para la investigación regional, donde la región (natural, geográfica, económica) es el marco de referencia idóneo y unidades básicas para la docencia donde zonas y dominios, en virtud de su carácter global, son adecuadas para comprender la distribución de los fenómenos en la superficie terrestre y las relaciones entre las distintas unidades.

La diferenciación de unidades de la superficie terrestre realizadas según criterio natural se caracteriza por los siguientes rasgos fundamentales:

- Carencia de universalidad. Cada clasificación se realiza para unos fines (climáticos, biogeográficos, geomorfológicos) y se ajusta a un criterio siendo prácticamente imposible la comparación entre ellas.

- Estabilidad. Al contrario de lo que ocurre en las clasificaciones regionales realizadas con criterios económicos o político-administrativos, las regiones de tipo natural no están sujetas a cambios coyunturales y presentan mayor duración en el tiempo.

- La nitidez de los límites entre unidades se difumina a medida que se aumenta la escala de análisis. Así, lo que son fronteras lineales bien definidas en las unidades de análisis de menor extensión (región a geotopo) se transforma en franjas de transición y solapamiento en los dominios y zonas.

\section{El clima: factor de diferenciación espacial}

La descripción de diferencias espaciales en el globo terrestre ha preocupado desde época griega. La explicación de la distribución de tierras y mares y en aquéllas de la diferente ocupación humana entre unos y otros lugares se encuentra en los textos clásicos, desde el s. V. a. J. C. En ellos es común el recurso a los rasgos básicos del clima, es decir, diferencias de temperatura e insolación, como factor que explica las diferencias entre los diversos ámbitos terrestres. Recordemos que el origen etimológico de la palabra clima («inclinación») se relaciona con la distinción de zonas con igual recepción lumínica solar. El manejo del factor climático como elemento fundamental de diferenciación territorial se mantendrá, invariablemente, hasta el s. XIX, cuando la constitución científica de la climatología (aparición del concepto de clima físico), amplíe los horizontes de análisis de esta rama geográfica por un lado, y las mejoras técnicas y la consolidación de sucesivos paradigmas geográficos, de raíz filosófica, conviertan, progresivamente, al clima en factor condicionante pero no determinante en la explicación de las diferencias regionales del mundo.

\section{2.a. Antecedentes remotos: Las zonas y los «klimata» griegos}

Los textos clásicos de la antiguedad griega manifiestan una distinción evidente entre «mundo habitable» y «mundo habitado», es decir, entre la totalidad del planeta, tal y como entonces se concebía, y el oikumene o tierra conocida. En la divisiones establecidas en uno y otro, el factor climático (básicamente, la insolación y las temperaturas) resultará capital. $\mathrm{El}$ «mundo habitable» se dividirá en «zonas» atendiendo a las diferencias térmicas, mientras que en el oikumene se establecerán «klimata» basadas en la diferente recepción lumínica que impone la latitud.

La disivión del mundo «habitable» se establecerá en función de dos criterios:

- la diferencia de temperatura entre el Ecuador y los Polos.

- la habitabilidad, es decir, existencia o no de vida humana. 
Así, se realizarán divisiones del mundo en zonas térmicas o grandes franjas más o menos cálidas cuyos límites van a variar entre unos y otros autores, de ahí que se acuda al criterio de la habitabilidad, es decir, de la existencia de vida humana, para matizar la ubicación precisa de la fronteras entre las diversas zonas. Aunque con el paso del tiempo, diversos autores matizarán esta cuestión, se mantenía la creencia de que únicamente la zona templada (que coincidía con el mundo conocido) era habitable.

La primera división del mundo habitable en zonas, manejando el criterio térmico, se atribuye, según Posidonio, a Parménides de Elea, hacia el 500 a. J.C. Parménides, continuador de la hipótesis jonia de que «la Tierra entera tiene forma de esfera al igual que el Universo» ${ }^{10}$, dividió la Tierra en cinco zonas desiguales: una tórrida, entre el trópico de verano y el trópico hiemal; dos templadas, entre el círculo ártico y el trópico estival y entre el tropico hiemal y el círculo antártico, respectivamente, y dos frías, entre los polos y las círculos árticos y antárticos respectivos. Parménides dio a la zona Tórrida el doble de anchura que el resto de franjas puesto que la extendió más allá de los Trópicos sobre las zonas Templadas. Cuestión explicable por la carencia, entonces, de noticias precisas sobre existencia de pobladores al sur de Egipto y Etiopía. Parménides caracterizó las condiciones térmicas de cada zona. Así, la zona tórrida central era, según su creencia, inhabitable por su excesivo calor; las dos zonas templadas disfrutaban de un clima apacible pero con sensibles oscilaciones térmicas estacionales y, las zonas frías se caracterizaban por la existencia de frío y nieve.

Con posterioridad, Aristóteles matizará esta división al hacer coincidir los límites con las líneas maestras, es decir, los dos círculos polares, situados a $54^{\circ}$ de latitud, y los dos trópicos, a $24^{\circ}$ de latitud. Para Aristóteles, la zona tórrida es, pues, la situada entre los trópicos. En la Meteorología (II,5. 362 a), defiende como habitables únicamente las dos zonas templadas. La vinculación entre hechos astronómicos y climáticos y la habitabilidad de las zonas será una constante en la Antigüedad.

Polibio (208-126 a. J.C.) establecerá una matización a esta división en 5 zonas al señalar la conveniencia de dividir la zona Tórrida en dos a través de la línea Ecuatorial. Estrabón criticará esta división por innecesaria «pues la simetría de las dos secciones de la zona Tórrida que produce el Ecuador y el estar situadas juntas muestra que este corte es superfluo e inútil» ${ }^{11}$. Una matización importante será, por su parte, la defensa de la habitabilidad de la zona tórrida, puesto que para Polibio, siguiendo el criterio de Eratóstenes, la «zona situada bajo el Ecuador es templada» ${ }^{12}$ por su mayor altitud, lo que le permite recibir más lluvias $\mathrm{y}$, en suma, acoger pobladores.

Posidonio (135-50 a. J.C.), criticará, por innecesaria, la división de la zona tórrida en dos secciones de Polibio y mantendrá la división clásica en 5 zonas. No obstante, como Polibio, rechaza el empleo de los Trópicos como límite de la zona habitable «como se desprende de los etíopes que viven más allá de Egipto». Ahora bien, contradice a Polibio en su argumento de la mayor altitud de la zona situada bajo el Ecuador, pues según Posidonio «en una superficie esférica no hay ninguna elevación debido a que es lisa». Para Posidonio la región situada bajo el Ecuador es «una gran llanura uniforme aproximadamente al nivel del mar» ${ }^{13}$.

Posidonio ensaya un nuevo método para delimitar las zonas a partir del empleo del gnomon. Según este sistema es posible distinguir: una zona entre los trópicos en que el

10 Vid. Estrabón. Geografía. (Libro II, cap. 2. 1.).

11 Vid. Estrabón. Geografía. (Libro II, cap. 3. 2.).

12 Vid. Estrabón. Geografía. (Libro II, cap. 3. 2.).

13 Vid. Estrabón. Geografía. (Libro II, cap. 3. 3.). 
gnomon proyecta sombra a ambos lados; dos zonas de una sola sombra entre el trópico y el Círculo Polar y dos zonas de sombra circular desde el Círculo Polar al Polo. Posidonio añadirá a estas 5 zonas «astronómicas» dos zonas más estrechas bajo los Trópicos «en las que aproximadamente la mitad de un mes el Sol está en su cénit y están divididas en dos por los Trópicos» ${ }^{14}$. Corresponden con las áreas áridas o desérticas que hoy conocemos «que tienen la particularidad de que son especialmente resecas, arenosas y estériles, excepto en silfio y algunos frutos ardientes y recocidos porque no hay cerca montañas que hagan que las nubes dejen caer sus lluvias ni tampoco están surcadas por ríos» ${ }^{15}$. Posidonio contrapone estas dos franjas con las zonas tropicales que tienen «una atmósfera más templada y una tierra más fértil y mejor regada ${ }^{16}$. Así pues, junto a las 5 bandas terrestres de origen astronómico-térmico, Posidonio distingue 7 bandas climático-biológicas al precisar las características de las zonas áridas insertas en la banda tórrida planetaria.

Geminos mantendrá el criterio de habitabilidad de la zona tórrida defendido por Polibio y Posidonio argumentando, en su Tratado, la existencia de pueblos etíopes en la extremidad de la zona tórrida e indica que si la zona tórrida se define por su inhabitabilidad, ésta no debe estar límitada por las líneas de los trópicos.

Estrabón (64 a. J.C.-23 d. J.C.) será partidario de la división del mundo habitable en 5 zonas establecidas a partir de las líneas mayores (trópicos y círculos polares). Para Estrabón la división en 5 zonas se justifica física y geográficamente. Físicamente porque está de acuerdo con los fenómenos celestes (con la división según las sombras — vid. supra-) y con las condiciones atmosféricas, puesto que según Estrabón «porque al juzgar las condiciones de la atmósfera con relación al Sol hay tres diferencias generales y fuertemente marcadas respecto a la disposición de animales y plantas y de todo cuanto vive bajo el aire o con él: el exceso de calor, su falta y el término medio». Por su parte, la división en 5 zonas se justifica geográficamente puesto que para Estrabón «la geografía se ocupa de delimitar la sección habitada por nosotros de una de las zonas templadas» y para remarcar la ausencia de habitabilidad en el resto de zonas «es necesaria la división en cinco zonas... las regiones situadas en el Ecuador y en la Zona Tórrida son inhabitables por el calor, las situadas en el Polo, por el frío, y las intermedias son templadas y habitables». Ahora bien, Estrabón no asigna límites precisos a estas zonas puesto que «no hay que delimitar lo invariable con marcas que varían». Así, rechaza la ubicación de la línea de los Trópicos manejada por sus predecesores Eratóstenes e Hiparco, y propone una nueva posición: el límite sur de la Zona Templada estará en el País de la Canela, 8.000 estadios al sur del Trópico de Cáncer y 8.800 al norte del Ecuador, mientras que el límite norte estaría en Yerne, a $54^{\circ}$ (como afirmaba Aristóteles).

Por su parte, el mundo conocido — oikumene — será distribuido en la antigüedad en una serie de franjas o bandas paralelas al Ecuador: los «klimata». Los «klimata» se definen como la porción del oikumene delimitada por dos paralelos en cuyo interior se incluyen ciudades, accidentes geográficos, pobladores de diversa etnia, etc. Es una forma más exacta de dividir la superficie terrestre mediante un criterio de tipo físico. En efecto, de nuevo, un elemento climático (la altura del sol) justifica las divisiones del espacio geográfico. En las «tablas de climas» de la antigüedad, una localidad se definirá, en primer lugar, por su pertenencia un «clima».

De entrada, hay que realizar una consideración sobre el concepto de «klimata» griego, puesto que se suele relacionar con aspectos exclusivamente astronómicos o cartográficos,

14 Vid. Estrabón. Geografía. (Libro II, cap. 2. 2.).

15 Vid. Estrabón. Geografía. (Libro II, cap. 2. 2.).

16 Vid. Estrabón. Geografía. (Libro II, cap. 2. 2.). 
es decir, posición de un punto en relación con la altura del sol, rechazando así el vocablo «kline» griego como origen del término clima, tal como se empleará desde el s. XVIII. Empero, en los textos clásicos, y con posterioridad, es común el empleo de la voz «klimata» o clima en su doble sentido de latitud y clima —atmosférico-. De hecho ambos aspectos están estrechamente relacionados: puesto que las diferencias de latitud suponen diferencias térmicas entre el Ecuador y el Polo. En efecto, la pertenencia a una banda climática («klimata» astronómico o cartográfico) supone la recepción de una cantidad de radiación solar determinada que, en suma, supone unos rasgos singulares de su medio ambiente atmosférico (clima atmosférico).

Por su parte, algún autor ha rechazado el carácter climático de las tablas de «klimatas» cuya finalidad se reduciría a la de mero instrumento cartográfico para la organización de las descripciones del mundo conocido. Estas divisiones del oikumene elaboradas por los clásicos a partir de supuestos astronómicos no tuvieron, en efecto, una intencionalidad climatológica en su sentido actual pero en las descripciones que realizan de cada uno de los «klimata» del mundo conocido se justifica su delimitación, es decir, el trazado de las líneas-frontera entre ellos, y se relatan aspectos atmosféricos que son propios de cada uno.

Cada «klimata» viene denominado con el nombre de la ciudad más importante conocida contenida en su interior; además, aunque la descripción de los aspectos geográficos de cada «klimata» varía de autor a otro, se pueden señalar como rasgos comunes la inclusión de una descripción de los límites naturales, de ríos, mares, montañas, etnias y productos naturales aprovechados por el hombre.

Estrabón atribuye la paternidad de las divisiones en «klimata» al matemático Eudoxo de Cnido (395-342 a. J.C.), quien en su «Descripción de la Tierra» realiza, en opinión de Pédech (1976), la primera descripción regional de corte naturalista del oikumene. Pero serán Eratóstenes e Hiparco los que porporcionarán las primeras tablas de «climatas» conocidas que serán manejadas, con sucesivas modificaciones, por diversos autores hasta la edad moderna. Eratóstenes (273-192 a. J.C.) calculó las proporciones del mundo conocido en 38.000 estadios de ancho dividiéndolo en 7 zonas (klimatas) de proporciones variables; de sur a norte, costas de Aromates, Meroe, Siena, Alejandría, Helesponto, Boristhene y Thule. Eratóstenes situará el límite norte del mundo conocido en al paralelo de Tule $\left(66^{\circ}\right)$. Hiparco (194-120 a. J.C.) modificará los cálculos del mundo conocido de Eratóstenes y en su «Memoria contra Eratóstenes», utilizando criterios astronómicos, presenta una división del oikumene en 11 «climas» de anchura regular ( $1^{\circ}$ ó 700 estadios). Para Hiparco un «clima» será el espacio comprendido entre $2^{\circ}$ de latitud. Los 11 climas en que se divide la anchura de 25.300 dada por Hiparco al oikumene son: Meroe, Siena, Alejandría, Babilonia, Sidon, Rodas, Atenas, Alejandría, Bizancio, Boristhene e Irlanda. Los límites meridional y septentrional del mundo conocido se sitúan en el Ecuador y a $61^{\circ} 10^{\prime}$ (Tule), respectivamente.

La división en climas Hiparco será utilizada, aunque con algunas modificaciones, años después, por Estrabón quien en la descripción de los «klimata» contenida en el libro II de su Geografía afirma que «hay que actuar sobre todo como lo hizo Hiparco» ${ }^{17}$. Estrabón reconoce asimismo 11 «climas», pero al no admitir, como tarea del geógrafo, la descripción de lugares desconocidos y rechazar la existencia de pobladores en las proximidades del Ecuador, sitúa el límite meridional del oikumene en el País de la Canela (12 $30^{\prime}$ norte $)^{18}$. Asimismo sitúa el límite septentrional del mundo conocido en Yerne (54 $4^{\circ}$ norte),

17 Vid. Estrabón. Geografía. (Libro II, cap. 5.34.).

18 Estrabón, como Homero y Eratóstenes, rechaza las pretensiones de Eudoxo de Cício de una tierra habitada al sur del Ecuador. Para él el oikumene ocupa únicamente la mitad del Hemisferio norte. 


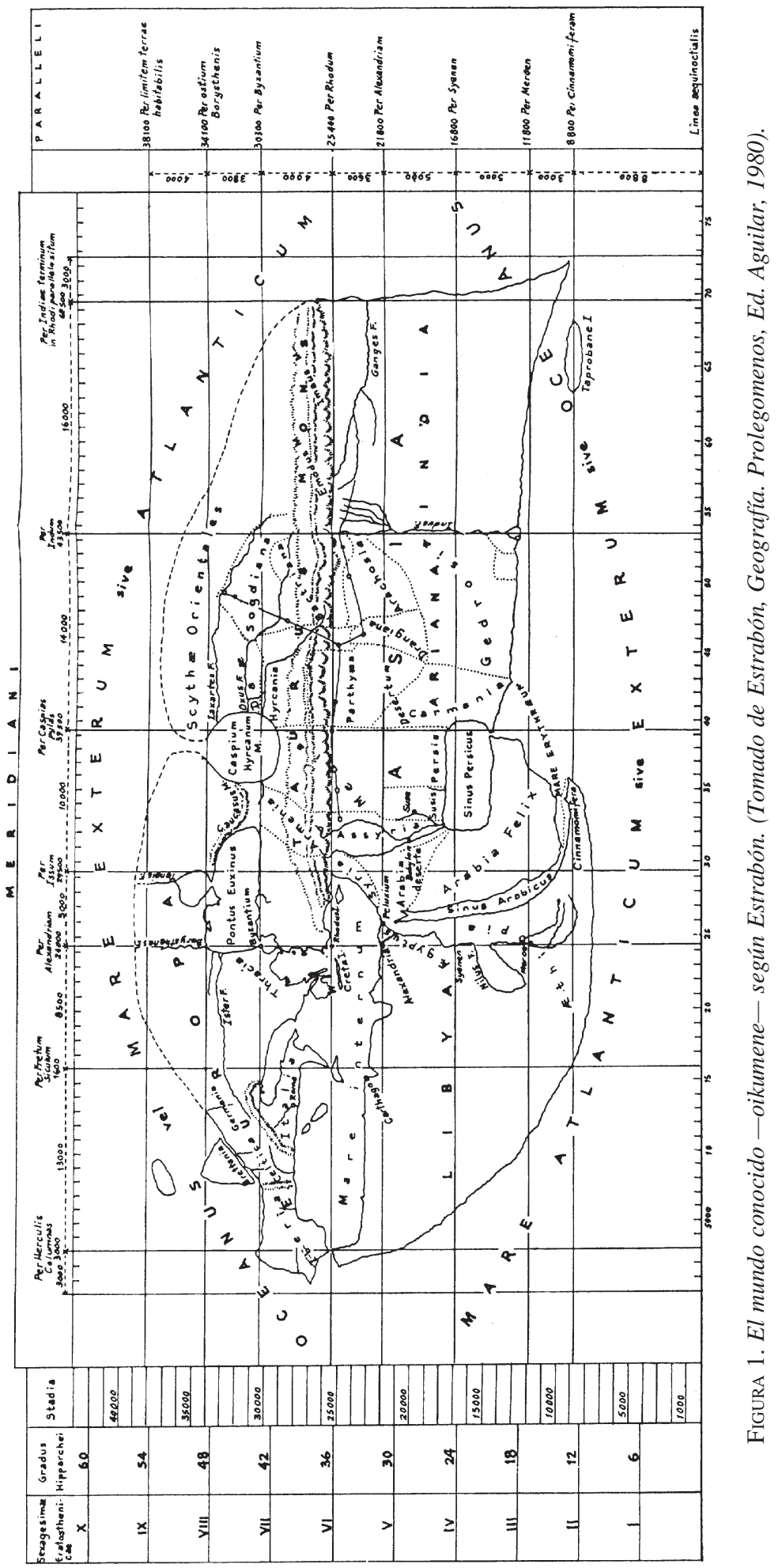


que coincide con el límite de la zona templada admitido por Aristóteles y Polibio (vid. supra). Entre ambos climas y en una anchura de 29.300 estadios se disponen de sur a norte Meroe, Siena, Bajo Egipto, Sidón, Rodas, Alejandría, Bizancio, Ponto, Borístenes, Meótide. El paso de un clima a otro está en función del día más largo cuya duración varía, con la latitud, entre 1 hora y 1/4 de hora. Los libros III a XVII de la Geografía están consagrados a la descripción del mundo conocido (vid. figura $\mathrm{n}^{\circ} 1$ ).

Claudio Ptolomeo matizará, en el s. II de nuestra era, la división del mundo conocido en su Introducción a la representación de la Tierra. Ptolomeo dividirá la Tierra (H. norte) en 26 líneas equidistantes a partir del Ecuador de oriente a occidente, desde la primera situada bajo la línea equinoccial donde el día es de 12 horas hasta la vigésimo sexta situada cerca de Escocia donde el día es de 18 horas y media. Ahora bien, el oikumene, que ocupa una cuarta porción del mundo habitable, es posible dividirlo en 7 climas principales en los que el paso de uno a otro supone la ganancia de media hora en el día más largo del año (solsticio de verano). Como para Estrabón, los climas vienen delimitados por un criterio «horario» y por tanto el intervalo latitudinal que ocupan varía, siendo menor conforme aumenta la latitud. A cada uno de ellos se asigna el nombre de una ciudad o accidente geográfico significativo anteponiendo el prefijo día (a través de); así se distinguen: Diameroe, Diasenes, Dialexandria, Diarodas, Diaroma, Diaborístenes y Diarifeos.

La obra de Ptolomeo tendrá gran repercusión en los siglos posteriores. Los tratados de geografía de época árabe, medieval y moderna contendrán continuas referencias a la «Geografía» de Ptolomeo y los planisferios reproducirán la división en «climatas» del mundo conocido.

Así pues, las descripciones del mundo de la antigüedad conceden un papel decisivo al clima como factor de diferenciación espacial. Se reconoce, asimismo, las influencias del clima en la naturaleza de los suelos (fertilidad, aridez), en la distribución de especial vegetales y animales y en los hombres y sus actividades (reparto de etnias, explotación de recursos agrarios, en suma, modos de vida).

Los tratados geográficos clásicos completan la descripción del mundo conocido con una división en 3 partes de criterio natural (Asia, África y Europa), distinguiendo en cada una de ellas «regiones» político-administrativas (países, reinos). Este método se mantendrá hasta la edad moderna, sin más cambios que los que impongan las modificaciones derivadas del descubrimiento progresivo de nuevas tierras.

\section{2.b. Divisiones del mundo en las edades media y moderna basadas en el factor climático}

En la Edad Media se mantendrá la división del mundo habitable y del mundo conocido en zonas y klimata respectivamente. En época árabe, los trabajos de los sabios Avicena, Albumasar y Alfragano introducen ligeras modificaciones a las tesis dictadas por los griegos sobre la superficie terrestre. Avicena defenderá el carácter templado del ecuador y por tanto su habitabilidad. Por su parte, los astrónomos Albumasar y Alfragano, continuadores de la idea ptolomeica de división del mundo conocido en 7 climas, equipararán a éstos con los siete planetas (Sol, Saturno, Júpiter, Mercurio, Venus, Luna y Marte), siguiendo antiguas creencias persas. Además Alfragano calculará, por vez primera, la superficie de cada clima en millas (un grado tiene 56 millas y $2 / 3$ ).

Los trabajos de los geógrafos árabes mantienen la división del mundo conocido de la antiguedad griega. Así, Ibn Khaldun, Idrisi y Aledrisen en sus descripciones geográficas utilizan la división en klimata. Como afirma Saavedra uno de los puntos de analogía entre Idrisi y los griegos es la división del mundo en siete franjas paralelas al Ecuador. Al-Idrisi en el prefacio de su tratado geográfico-descriptivo señala que su labor era describir las 
ciudades y territorios representados en el planisferio, el tipo de agricultura y poblaciones, los rasgos del medio físico, las costumbres, curiosidades y religiones de las gentes que habitan cada uno de los siete climas en los que dividía el mundo. Por su parte, Ibn Khaldun, en su Mukaddimah, utiliza también la división del área habitada (cultivada) en siete climas, observando que las áreas más septentrionales y más meridionales están menos pobladas. Para Ibn Khaldun «la civilización encaja entre las zonas tercera y sexta» y añadía que el clima ejerce una importante influencia en el carácter de las gentes: las personas que viven en las regiones más cálidas y costeras del sur son más alegres, mientras que los habitantes de las regiones frías, montañosas y accidentadas con más tristes y melancólicas. No obstante, hay que señalar que los geógrafos árabes presentan otra novedad en la utilización del concepto «clima» al utilizar la expresión clima (iklim) con el doble significado de clima-inclinación de rayos solares (klimata griego) y como división administrativa. Josef Antonio Conde, traductor, en el s. XVIII, de la «Descripción de España» de Xeris Aledris hace notar esta doble acepción de la expresión clima al afirmar que dicho autor «maneja la división del mundo conocido en 7 climas «fijos y verdaderos», pero supone otros en cada región, que contienen varias provincias, dándoles la extensión que le pareció más cómida». Habla de clima verdadero (los klimata) o de clima conocido (división administrativa, provincia) «el verdadero es uno de los siete, y el conocido aquella extensión arbitraria de país que facilita sus divisiones». Un ejemplo de ello se expone en la misma descripción de España de este autor cuando, tras indicar que las tierras ibéricas se incluyen en el $4^{\circ}$ clima verdadero, distingue dentro de él numerosos climas conocidos: «alinda el clima Xêduna y es del clima Albuhiret por el norte; y en él entre otras ciudades Medina Esbila, y Medina Carmûna, y Alxêna, y muchos castillos; y alinda el clima Alxarf... Todos ellos son los climas de Esbania, llamada propiamente Andalus».

Una cuestión debatida en época bajomedieval será la porción de la tierra habitable y la habitabilidad de la zona tórrida. Sobre la primera, la lectura del tratado Sobre el cielo y el mundo de Aristóteles, en el que se afirma, en contra a la opinión de Ptolomeo, que es habitable más de un cuarto de la superficie terrestre irá ganando adeptos, de ahí que se fueran precisando la forma y extensión de diversas partes del mundo conocido, sobre todo de los mares circundantes, reduciendo el excesivo tamaño que se había concedido hasta entonces al mar Mediterráneo y al mar Océano. Por su parte, con el paso del tiempo irá aceptándose la posibilidad de vida en la zona tórrida «al sur de los desiertos», cuestión que terminará admitiéndose en el tránsito de los ss. XV a XVI en la época de los grandes descubrimientos (vid. infra).

Todas estas cuestiones encuentran en el Ymago Mundo del erudito religioso Pierre d'Ailly, —el «hombre más sabio de todos los de su tiempo» ${ }^{19}$ —, una brillante compilación. Para la división del mundo habitable, D'Ailly defiende la división de la tierra en 5 zonas (cap. VI) e indica que la primera y la última de ellas — las dos zonas polares - son «inahabitables por culpa del mucho frío» (vid. figura $\mathrm{n}^{\circ} 2$ ). Sobre la habitabilidad de la zona tórrida no es tan concluyente al indicar que «se la tiene por inhabitable a causa de su excesivo calor». D’ Ailly dedica el capítulo 7 de su tratado ( «Diversidad de opiniones sobre la habitabilidad de la Tierra») a presentar un conjunto de consideraciones «evidentes» sobre la templanza de aquella zona y la posibilidad de vida al sur de los desiertos. D'Ailly, siguiendo opiniones de diferentes autores, indica que «se ha demostrado que no solamente están habitados los siete climas, sino que hay poblaciones humanas en una cuarta parte de la tierra y aun más».

19 Vid. Prefacio del Ymago Mundi a cargo de Juan de Westfalia (1483). Biblioteca Colombina de Sevilla. 


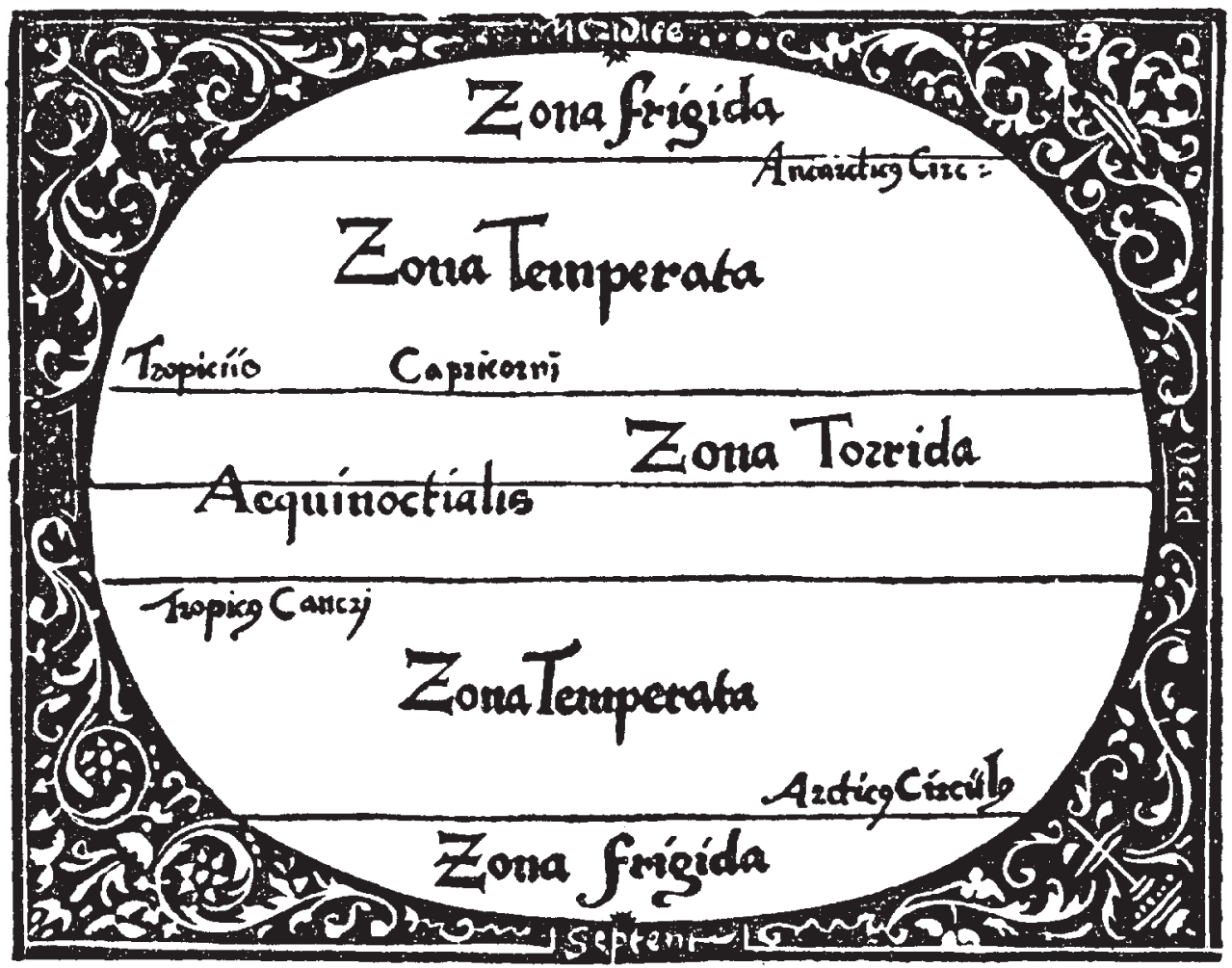

FIGURA 2. División del mundo habitable en «zonas climáticas».

En la división del mundo conocido, D'Ailly maneja el concepto de klima utilizado por los astrologos árabes Albumasar y Alfragano - heredado a su vez de los griegos-, es decir, el espacio de la tierra donde el día más largo sobrepasa en media hora al día más largo de la otra parte de la tierra, o resulta sobrepasado en ese mismo tiempo y, siguiendo a Ptolomeo, divide el mundo habitado en siete climas (diaMeroe, diaSenes, diaLexandria, diaRodas, diaRoma, diaDoristenes y diaRifeos). D'Ailly incluye dos conceptos para denominar los espacios supuestamente habitables situados más alla del mundo conocido. Así, las zonas «habitadas» situadas más alla de la primera línea son denominadas anteclimas (Ptolomeo ya había manejado esta denominación en el s. II) y a las que están más allá del séptimo clima, postclimas. Además, D’Ailly presenta las ideas de Ambrosio en su Hexameron sobre la nomenclatura de los hombres en función de la sombra que proyectan ${ }^{20}$ : esciápodes o sin sombra puesto que están sin sombra dos días al año en las partes del meridiano donde, «por tener el sol en sus cabezas, quedan iluminados por todo alrededor» $\mathrm{y}$ anficios o «sombreados» que viven en el Ecuador.

En el capítulo décimo tercero del Ymago mundi, D’Ailly recoge las opiniones de diferentes sabios de la antigüedad sobre la diferencia de los lugares y las diversas condiciones de habitabilidad. D'Ailly indica como Aristóteles, Ptolomeo, Plinio y Halli relacionan la existencia de hombres sabios con la pertenencia a la parte central de la zona habitada

20 Vid. supra, ideas de Posidonio sobre la diferente sombra existente en las zonas terrestres. 
(climas $3^{\circ}, 4^{\circ}$ y $5^{\circ}$ ). Por lo demás, D'Ailly dedica lo esencial de su Ymago mundi a la descripción del mundo conocido organizando ésta en tres partes (Asia, África y Europa). Cada región, nación o lugar viene caracterizado por su pertenencia a un clima y su posición exacta se detalla en el tratado de Cosmografía de este autor ${ }^{21}$.

La división del mundo conocido en «klimata» será común en las obras editadas en las postrimerías de la época medieval. Baste citar, al respecto, la inclusión de una «tabula climatum» en el códice astrológico del cuatroccento italiano la «Sphaerae coelestis et planetarum descriptio» ${ }^{22}$. Hay que señalar que en este códice se contienen una serie de indicaciones sobre la desigual duración de los días, —recogidas por D’ Ailly en el Epílogo de la Mapamundi ${ }^{23}$ - , en las que se completa la tabla de duración de los días del oikumene hasta el Polo. Manejando criterio astronómico, se señala cómo en el Círculo ártico el día es de 24 horas en el solsticio de verano y desde allí hasta el Polo es posible distinguir 6 climas de mes, es decir, franjas donde el día dura un mes, dos meses, etc. hasta el Polo donde «la mitad del año completo es un solo día artificial y la otra mitad sólo una noche» (6 meses). Así pues, desde finales de la edad media las tablas de «climas» del orbe terrestre presentan no sólo los 7 climas del oikumene, con indicación de la duración del día más largo (solsticio de verano) sino la relación total de climas horarios posibles hasta el Círculo Ártico (ganancia de 1/2 hora) hasta completar un día (24 h.) y desde allí hasta el Polo la existencia de 6 climas de «mes». En total, las tablas de «climas» contendrán 23 climas semihorarios (los 7 primeros incluyen el oikumene) separados por 24 líneas maestras y 6 climas de mes.

El tránsito de los siglos XV a XVI conocerá una revisión de la teoría de las zonas. En efecto, una serie de tratados cuestionarán decididamente el dogma de la inhabitabilidad de las zonas tórridas y polares. Esta idea, presente ya como conjetura en algunos escritos clásicos y medievales (vid. supra), se conforma como evidencia demostrable a raíz de los nuevos descubrimientos geográficos llevados a cabo por portugueses y españoles. Como indica Randles (1980) en el intervalo de cuarenta años, —entre el tratado In Manilium commentum del italiano Lorenzo Bonicontri (1484) y la Suma de Geographia del español Martín Fernández de Enciso (1519)—, se produce la aceptación de la habitabilidad de las zonas tórridas y polares. Una serie de tratados ${ }^{24}$ publicados en dicho lapso de tiempo irán presentando noticias sobre la existencia de habitantes en ambas zonas (sobre todo en la tórrida), evidencia que se mantendrá, sin apenas alteración ${ }^{25}$, durante toda la edad moderna.

Las obras geográficas de la edad moderna, fieles a la estructura en dos partes consolidada en el s. XVI que incluye una parte general - Geografía General—y una regional - Geografía Regional o Especial—, incluirán divisiones del mundo en zonas y climas, manteniendo la tradición clásica que tendrá amplia difusión desde la traducción al latín de la obra de Ptolomeo (1410). Pueden citarse, al respecto, los trabajos de F. Clüver (Intro-

21 Pierre D’Ailly escribe dos compendios de cosmografía (I y II) entre 1412 y 1414 para aclaración y suplemento — sic — de los tratados primero y octavo de su Ymago mundi dedicados a explicar la división y las partes del orbe terrestre, a semejanza, en suma, de la obra de Ptolomeo.

22 Este manuscrito miniado, de origen lombardo, se atribuye a Cristoforo De Pedris, en la sexta-séptima década del siglo XV.

23 Vid. Piere D’Ailly Ymago Mundi, (Epílogo de la Mapamundi, Alianza Editorial, 1992, pp. 152-155). Este epílogo es una descripción de la figura del mundo que, como señala el autor, completa el Ymago mundi.

24 Baste citar las obras de Jerome Münzer (Itinerarium, 1495), Diogo Gomes (finales s. XV), Pedro Ciruelo (Uberrimum Spherae Mundi Comentum..., 1498) y Duarte Pacheco Pereira (Esmeraldo de Situ Orbis, 1508).

25 Únicamente, a finales del s. XVI, y como consecuencia de la contrarreforma, la obra de Tommaso Bozio (1592), volverá a defender la inhabitabilidad de la zona tórrida, para preservar el honor de la cultura antigua y la autoridad de la Biblia. 
ductio in Universam Geographiam, 1624) y de Ph. Briet (Parallela Geographiae veteris et novae, 1648); en ellos, la descripción de las teorías de zonas y climas, se insertan en la parte «general» o «astronómica» de la geografía, ocupando, un reducido espacio respecto al volúmen global de las obras. Además, la descripción posterior de regiones que se presenta en los capítulos de la parte «regional» no se realiza en función de la división en climas. Algunos años antes, el padre José de Acosta en su «Historia Natural y moral de las Indias» (1590), a la vista de los descubrimientos realizados en el Nuevo Continente, había aclarado las ideas sobre la naturaleza de la zona Tórrida, desmintiendo creencias mantenidas desde época griega, sobre la penuria de lluvias, aguas, pastos y exceso de calor de esta zona.

En el crepúsculo de la edad moderna, la magna «Geografía General» de Bernhard Varenio, dedica dos capítulos del Libro II («Parte Relativa. Acerca de las propiedades celestes de la Tierra») a explicar la división de la Tierra en zonas (cap. XXIV) y la división de la superficie en climas (cap. XXV), respectivamente. Varenio, conocedor de la tradición clásica y seguidor de la influencia tolemaica, realiza una revisión de las teorías de división de la tierra defendidas por diversos autores, desde la antiguedad griega hasta el s. XVII. En el esquema previsto para la descripción de las regiones del orbe de su inconclusa «Geografía Especial» la pertenencia a una zona y un clima se incluye entre los «factores celestes» que caracterizan una región en los que se contempla asimismo la explicación del «calor y meteorología del año, vientos, lluvias y otros fenómenos atmosféricos». Se muestra así la estrecha relación entre la pertenencia de un lugar a una zona o clima y los rasgos específicos que presenta el medio ambiente atmosférico en dicha posición geográfica.

El tránsito del s. XVIII irá marcando importantes modificaciones en los estudios del medio natural, que sentarán las bases para la renovación metodológico-conceptual de la geografía del s. XIX. Para la disciplina climática el s. XVIII es el siglo de la aparición de instrumental meteorológico (termómetros, barómetros, anemómetros, higrómetros) y de la consolidación de las primeras redes de observación meteorológica en Europa, aspectos ambos de importancia capital para el establecimiento de los estudios «científicos» de climatología («clima físico») en el s. XIX.

En ausencia de obras geográficas de relevancia en este siglo, tras la publicación de la Geografía de Varenio, «el edificio (pensamiento geográfico)... fue invadido por los adeptos de las ciencias vecinas» (Martonne, 1973 Tomo I, p. 30). No obstante, las aportaciones que se realizan desde las ciencias naturales y la filosofía van a resultar decisivas para la evolución posterior de la geografía. Así, dentro de lo que hoy se denomina medio ambiente climático, el s. XVIII viene marcado por la aparición de la teoría de los climas y el origen del ambientalismo, aspectos de amplia relevancia por el amplio número de partidarios y detractores que cultivaría.

Estas dos cuestiones encuentran su marco conceptual en las obras de Buffón y Montesquieu. En opinión de Urteaga (1993), la influencia del clima en la explicación de las diferencias humanas, políticas y culturales del orbe terrestre fue un asunto común entre los tratadistas europeos del s. XVIII, aunque nunca llegó a existir consenso completo sobre este aspecto. Tres campos centrarán lo esencial del ambientalismo o determinismo del factor climático en el s. XVIII: a) La teoría etio-patológica, que hunde sus raices en el legado hipocrático, según la cual las condiciones meteorológicas son uno de los factores determinantes de la salud humana y las diferentes características que aquéllas presentan en la superficie terrestre permite establecer categorías de salubridad de los distintos ámbitos geográficos. En este razonamiento se encuentra el origen de la geografía médica. b) La teoría de las razas, que explica la diversidad espacial de la especie humana en función de los rasgos que presenta el clima en cada lugar. Buffon en su Historia Natural, llega a 
afirmar que las razas no son una entidad esencial y definida, sino el producto superficial y mudable del clima y los modos de vida. c) Una reflexión histórico-política, que explica la diversidad cultural de las diferentes regiones del mundo como efecto de los rasgos climáticos de cada una de ellas. En El Espíritu de las Leyes, Montesquieu afirma que el clima gobierna temperamento y costumbres como argumento principal de la influencia del ambiente climático en la aparición y persistencia de formas culturales («las necesidades en los diferentes climas han dado origen a los distintos modos de vida»). Montesquieu dedica 4 libros de esta obra (XIV a XVII) a establecer las relaciones del clima con las leyes en general, con las leyes «de la esclavitud civil», con las leyes de la «esclavitud política» y con las de la «servidumbre política».

Aunque el ambientalismo y el determinismo climático será ampliamente discutido por numerosos filósofos de la Ilustración (Voltaire, entre ellos), lo importante es destacar el nacimiento ${ }^{26}$, en este momento, de una corriente de interpretación de las relaciones hombre-medio y de la diversidad de seres y lugares que mantendrá viveza hasta bien entrado el siglo XX.

Por lo demás, la obra cartográfica publicada en este siglo, al margen de la mejora de los sistemas de proyección y su cuidada elaboración siguen incluyendo como aspecto básico la división del Hemisferio norte en «climas» horarios (o de media hora) hasta el Círculo polar y 6 «climas» de mes desde allí hasta el Polo. Así puede contemplarse, entre otros, en el «Planisphaerium Terrestre cum utroque coeliesti Hemisphaerio, sive diversa orbis Terraquei» de Adam Zürner (1710). Algunos planisferios editados en la segunda mitad del s. XVIII aumentarán, como novedad, a 9 el número de climas o franjas de división del mundo conocido.

En el amplio período de tiempo que transcurre desde la época griega y el s. XVIII, el factor climático se presenta como el elemento básico para comprender la desigual distribución de los hechos geográficos en el conjunto del globo terrestre. La necesidad de sistematizar el conocimiento de la realidad conocida se traducirá en la aparición de las «teorías» de «zonas» y «climas». La distinta recepción de energía solar, traducida en las diferencias manifestadas en dos elementos climáticos básicos (temperaturas e insolación) está en la base de ambas. El clima aparece, a la vez, como elemento condicionador de la existencia de vida humana y como de explicar (cartografiar) la distribución de la misma en el orbe terrestre.

La evolución de ambas teorías será diversa. Así, mientras la división en «climas» irá perdiendo vigor tras la obra de Varenio, la división en «zonas» permanecerá hasta nuestros días, siendo la base de numerosas clasificaciones climáticas y de divisiones del mundo elaboradas en función del factor climático surgidas desde mediados del s. XIX. Recordemos, en este último caso, la consolidación, por ejemplo, de la geografía zonal en los años sesenta y setenta de nuestra centuria.

26 No hay que olvidar que el legado hipocrático y, en suma, el determinismo de la naturaleza sobre el hombre había permanecido en el Renacimiento a través de la obra de J. Bodin (Los seis libros de la República, 1580). 


\section{Bibliografía}

ALBENTOSA SÁNCHEZ, L. (1984): «La expansión del mundo conocido y la geografía durante el siglo XVIII» en El siglo XVIII. Aproximación interdisciplinar, Tarragona.

AUJAC, G. (1966): Strabon et la science de son temps, Societe d’edition «Les Belles Lettres», París, pp. $147-170$ y $267-273$.

BROC, N. (1980): La Géographie de la Renaissance (1420-1620), biblioteque Nationale, París, $261 \mathrm{pp}$.

CASTILLO REQUENA, J. M. (1991): Reflexiones sobre el tiempo y clima, Universidad de Granada y Diputación Provincial de Almería, Granada, 263 pp.

D’AILLY, P. (1992): Ymago mundi y otros opúsculos, Alianza Editorial, (Biblioteca de Colón, II). Madrid, $356 \mathrm{pp}$.

ESTRABON (1980): Geografía. Prolegómenos. (Traducción y notas por Ignacio Granero; Introducción por Arturo A. Roig). Ed. Aguilar, Madrid, 477 pp.

ESTRABON (1991): Geografía. Libros I-II. (Introducción general de J. García Blanco; Traducción y notas de J. L. García Ramón y J. García Blanco). Biblioteca Clásica Grados. Madrid, 559 pp.

MARTONNE, E. (1973): Tratado de Geografía Física. 3 vols. Ed. Juventud, Barcelona.

PEDECH, P. (1976): La géographie des grecs. P.U.F., París, 202 pp.

PEDELABORDE, P. (1982): Introducción a la climatología, Ed. Oikos-Tau, Barcelona, 144 pp.

RANDLES, W. G. L. (1980): De la Terre Plate au Globe Terrestre, Libraire Armand Colin, París, $119 \mathrm{pp}$.

RIBEIRO, O. (1987): Introduçao au estudo da geografía regional, Ediçoes Joao Sá da Costa, Lisboa, $148 \mathrm{pp}$.

SAAVEDRA, E. (1881): La Geografía de España del Edrisi, Imp. Fortanet, Madrid.

SZABO, A. y MAULA, E. (1986): Les débuts de l'astronomie de la géographie et de la trigonométrie chez les grecs, Librairie philosophique J. Vrin, París.

UNWIN, T. (1995): El lugar de la Geografía, Ed. Cátedra, Madrid, 342 pp.

URTEAGA, L. (1993): «La teoría de los climas y los orígenes del ambientalismo» en Geocrítica, $\mathrm{n}^{\circ}$ 99, Universitat de Barcelona, Barcelona, $55 \mathrm{pp}$.

VARENIO, D. (1650): Geografía General en la que se explican las propiedades generales de la Tierra. (Edición y estudio preliminar de Horacio Capel, 1974). Ediciones de la Universidad de Barcelona, Barcelona, 146 pp.

VIERS, G. (1981): Climatología, Ed. Oikos-Tau, Barcelona, 309 pp.

XERIF ALEDRIS (1799): Descripción de España (Traducción y notas de D. Josef Antonio Conde). Imprenta Real, Madrid, 234 pp. 